\title{
Fuzzy PID Control of Space Manipulator for Both Ground Alignment and Space Applications
}

\author{
Fu-Cai Liu Li-Huan Liang Juan-Juan Gao \\ Key Lab of Industrial Computer Control of Hebei Province, Yanshan University, Qinhuangdao 066004, China
}

\begin{abstract}
Considering gravity change from ground alignment to space applications, a fuzzy proportional-integral-differential (PID) control strategy is proposed to make the space manipulator track the desired trajectories in different gravity environments. The fuzzy PID controller is developed by combining the fuzzy approach with the PID control method, and the parameters of the PID controller can be adjusted on line based on the ability of the fuzzy controller. Simulations using the dynamic model of the space manipulator have shown the effectiveness of the algorithm in the trajectory tracking problem. Compared with the results of conventional PID control, the control performance of the fuzzy PID is more effective for manipulator trajectory control.
\end{abstract}

Keywords: Space manipulator, trajectory tracking, ground alignment, space applications, fuzzy proportional-integral-differential (PID), gravity environment.

\section{Introduction}

In recent years, the problems of trajectory tracking control of robot manipulator have drawn a great attention ${ }^{[1,2]}$. With the development of space industry, the space manipulator is playing an increasingly important role, such as constructing, on-orbit servicing and the maintenance of the International Space Station. For these applications, it is necessary to study the manipulator. Since the dynamic equations of the space manipulator are tightly coupled, highly nonlinear and uncertain, using the conventional (PID) control is difficult to achieve the desired tracking performance. Many control algorithms have been proposed to deal with the robotic control problems ${ }^{[3-6]}$. However, most studies on trajectory tracking of free-floating space manipulators have assumed that the kinematics are exactly known. A passivity based adaptive Jacobian controller was proposed for freefloating space manipulators whose kinematics and dynamics will change or are difficult to derive exactly ${ }^{[7]}$. Simulation results showed good performance of the proposed controller. In order to overcome the problem of the nonlinear model, the dynamics of the space manipulator can be derived using the dynamically equivalent model approach ${ }^{[8]}$. A stable adaptive fuzzy-based tracking control algorithm was developed for robot systems with parameter uncertainties and external disturbances ${ }^{[9]}$. The developed fuzzy adaptive robust controller can make the closed-loop robot system stable and the trajectory tracking performance guaranteed.

However, all the above researches were only for ground robots or free-floating manipulators. The whole process that from ground alignment to space applications was not taken into consideration. From ground alignment to space applications the dynamic model of the manipulator will change which may result in the change of its movement properties. Therefore, it is necessary to design a modelfree controller to make the robot system track the desired

Regular paper

Manuscript received January 10, 2013; revised August 2, 2013

This work was supported by National High Technology Research and Development Program of China (863 Program) (No. 2011AA). trajectory in different gravity environments.

Considering the problems above, a control algorithm is developed by combining the fuzzy approach with the PID control method. The conventional PID controller, due to its simplicity in structure and ease of design, has been widely used in industrial applications. But the weakness of the controller is that it largely depends on the parameters of the controlled object. The fuzzy controller is very suitable for a controlled object with nonlinearity and even with unknown structure ${ }^{[10]}$. If a problem is not well understood and cannot be precisely described mathematically, but has good direction on how to control it, a fuzzy logic controller often works well. However, the determination of the rules and definitions are not obvious for complex systems and it depends on people's experience too much. Therefore, the fuzzy PID control algorithm is developed by combining the strengths of the two algorithms. It has been a common practice to combine the fuzzy controller with the simple conventional controller PID ${ }^{[11]}$. So far, many fuzzy PID control theories have been introduced ${ }^{[12]}$. In [13], a new fuzzy PID was designed to control some known nonlinear systems. The fuzzy controller tuned with particle swarm optimization (PSO) also was researched ${ }^{[14]}$ and a new fuzzy auto-tuning position speed PID control strategy was proposed ${ }^{[15]}$, which can not only ensure a higher position accuracy but also keep the robot operating smoothly. In order to realize automatic regulating of PID parameters a fuzzy inference method was developed $^{[16]}$ and the applications of the controller in some systems ware studied with Matlab.

On this basis, this paper uses a fuzzy PID controller to complete the task of controlling the space manipulator to track a given trajectory for both ground alignment and space applications. The fuzzy rules are designed according to the condition of the space manipulator control system. Simulation results have shown that it can get a satisfactory control effect.

There are two contributions of this paper. First, a novel idea has been proposed to deal with the problem of the space manipulator aligned on the ground and applied in 
the space and the second is that the designed fuzzy PID controller can ensure that the space manipulator can get a good control effect for both cases.

The rest of this paper is organized as follows. Section 2 establishes the kinematics and dynamics model for the two conditions. Section 3 designs the fuzzy PID controller to control the space manipulator under two conditions. Section 4 shows the simulation results and compares the results with the conventional PID controller. Finally, conclusions are drown in Section 5.

\section{System description}

The space manipulator in this paper assumes the followings:

1) The system is considered as a rigid system.

2) In the space the micro-gravity is ignored, and the system is in a free-floating condition. And no external forces or torques apply on the system.

3) The system consists of a base and several links. The pose of the base is not controlled actively, and every joint between the links can rotate freely within a range under active control. The space manipulator system is shown in Fig. 1, where MC is the mass center of the system.

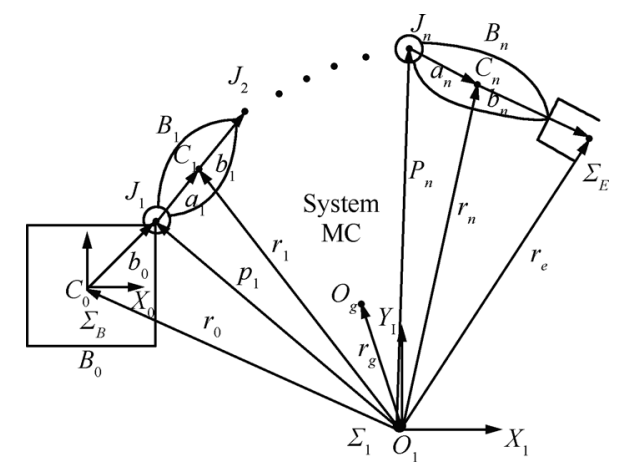

Fig. 1 The model of $n$-DOF free-floating space manipulator

\subsection{Ground state manipulator modeling}

When the space manipulator system is in the ground alignment stage the base can be fixed, for there exists gravity. To facilitate discussion, in this paper a planar 2-link manipulator is chosen, as shown in Fig. 2.

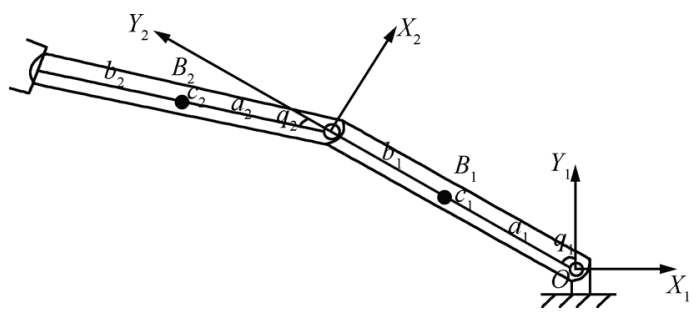

Fig. 2 The 2-link manipulator on the ground

In Fig. $2, B_{i}$ is the $i$-th link; $C_{i}$ is the centroid of the link; $m_{i}$ is the link mass; $\ell_{i}$ is the link length. $a_{i}$ is a scalar from the $i$-th joint to the centroid of the next link; $b_{i}$ is a scalar from the $i$-th centroid of the link to the joint of the next link; $q_{i}$ is the joint position.
By geometric analysis and using the homogeneous coordinate transformation, we get the position of the endeffector $^{[17]}$.

$$
\boldsymbol{p}_{e}=\left[\begin{array}{c}
-l_{1} s_{1}-l_{2} s_{12} \\
l_{1} c_{1}+l_{2} c_{12} \\
0
\end{array}\right]
$$

where $s_{1}=\sin q_{1}, s_{12}=\sin \left(q_{1}+q_{2}\right), c_{1}=\cos q_{1}, c_{12}=$ $\cos \left(q_{1}+q_{2}\right)$.

When (1) is differentiated, the end speed of the robot manipulator is available.

$$
J(q) \dot{q}=\dot{x}
$$

where $\boldsymbol{J}(\boldsymbol{q}) \in \mathbf{R}^{2 \times 2}$ is the Jacobian matrix related to the robot manipulator motion, and $\boldsymbol{q}=\left[\begin{array}{ll}q_{1} & q_{2}\end{array}\right]^{\mathrm{T}}$ is the joint angle vector and $\boldsymbol{x} \in \mathbf{R}^{2}$ is the end position.

For the planar 2-link robot manipulator, the dynamic of the space manipulator is derived using the Lagrange formulation.

$$
M(q) \ddot{q}+B(q, \dot{q}) \dot{q}+\boldsymbol{G}(\boldsymbol{q})=\tau
$$

where $\boldsymbol{M}(\boldsymbol{q}) \in \mathbf{R}^{2 \times 2}$ is the inertia matrix; $\boldsymbol{B}(\boldsymbol{q}, \dot{\boldsymbol{q}}) \in \mathbf{R}^{2 \times 2}$ is the vector of the Coriolis and centrifugal forces; $\boldsymbol{G}(\boldsymbol{q}) \in$ $\mathbf{R}^{2}$ is the gravity vector; $\tau \in \mathbf{R}^{2}$ is the joint torque.

\subsection{Free floating state manipulator mod- eling}

According to the properties that have been assumed previously, the free floating space manipulator is shown in Fig. 3. The freedom of the selected planar manipulator will be added to five for the space manipulator will rotate around its centroid and translate along the axis. When the main body of manipulator moves, a dynamic force or torque will apply on the base and the attitude and position of the base will change.

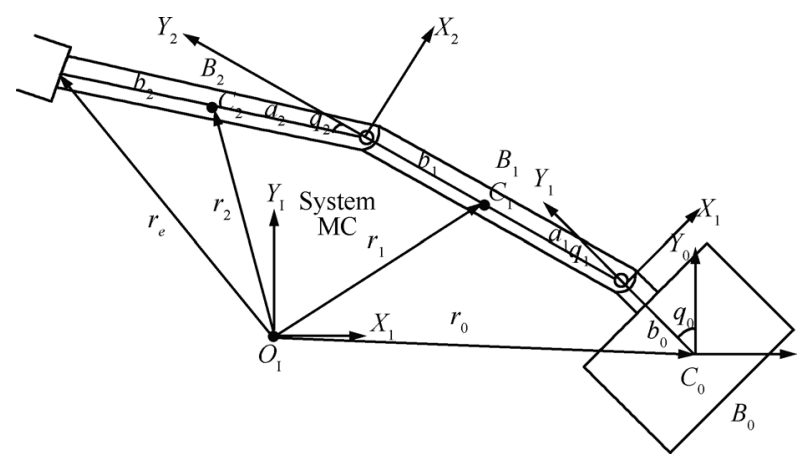

Fig. 3 The 2-link free-floating manipulator in space

In the figure, $B_{0}$ is the base of the space manipulator; $C_{0}$ is the centroid of the base; $m_{0}$ is the mass of the base; other symbols have been defined in Fig. 1.

The kinematic and dynamic equations can be established in the similar method of the ground case. The position of the end-effector is

$$
\boldsymbol{p}_{e}=\left[\begin{array}{c}
x_{c 0}-b_{0} s_{0}-l_{1} s_{01}-l_{2} s_{012} \\
y_{c 0}+b_{0} c_{0}+l_{1} c_{01}+l_{2} c_{012} \\
0
\end{array}\right]
$$


where $s_{0}, s_{01}, s_{012}, c_{0}, c_{01}, c_{012}$ have the same definitions as (1). When (4) is differentiated, the end $\dot{\boldsymbol{q}}$ velocity of the robot manipulator can be available.

$$
J^{*}(q) \dot{q}=\dot{x}
$$

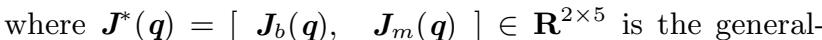
ized Jacobian matrix, $\boldsymbol{J}_{b}(\boldsymbol{q})=\partial x / \partial q_{b} \in \mathbf{R}^{2 \times 3}$ is the base Jacobian matrix, $\boldsymbol{J}_{m}(\boldsymbol{q})=\partial x / \partial q_{m} \in \mathbf{R}^{2 \times 2}$ is the robot manipulator Jacobian matrix and $\boldsymbol{q}=\left[\begin{array}{ll}\boldsymbol{q}_{b}^{\mathrm{T}}, & \boldsymbol{q}_{m}^{\mathrm{T}}\end{array}\right]^{\mathrm{T}}=$ $\left[x_{0}, \quad y_{0}, \quad q_{0}, \quad q_{1}, \quad q_{2}\right]^{\mathrm{T}} \in \mathbf{R}^{5}$ are the center of mass, the base attitude angle and each joint angle, respectively.

According to the Lagrange formulation the dynamic equation is established as

$$
\boldsymbol{M}(\boldsymbol{q}) \ddot{\boldsymbol{q}}+\boldsymbol{B}(\boldsymbol{q}, \dot{\boldsymbol{q}}) \dot{\boldsymbol{q}}=\left[\begin{array}{cc}
\mathbf{0}_{3 \times 1}^{\mathrm{T}}, & \tau_{2 \times 1}^{\mathrm{T}}
\end{array}\right]^{\mathrm{T}}
$$

where $\boldsymbol{M}(\boldsymbol{q}) \in \mathbf{R}^{5 \times 5}$ is the inertia matrix; $\boldsymbol{B}(\boldsymbol{q}, \dot{\boldsymbol{q}}) \in \mathbf{R}^{5 \times 5}$ is the vector of the Coriolis and centrifugal forces. To ensure that the system is stable, $\boldsymbol{M}(\boldsymbol{q}) \in \mathbf{R}^{5 \times 5}$ should be symmetric and positive-definite. The matrix $\boldsymbol{M}(\boldsymbol{q})-2 \boldsymbol{B}(\boldsymbol{q}, \dot{\boldsymbol{q}})$ can be skew-symmetric ${ }^{[18]}$.

\section{Controller design}

A fuzzy logic controller is normally built with four distinct components, namely fuzzification, inference engine, rule base and defuzzification ${ }^{[19]}$. A fuzzification unit is used to transform the input of the controller into a form which can be expressed by the rule base and also can be compared with the other rules. The defuzzification step is used to transform the conclusion derived from the inference into the input of the controlled objects. These two processes require heuristic rules and membership functions to encode the desired system. The basic configuration of a fuzzy system is a collection of the IF-THEN rules, where the $j$-th rule, is in the following form:

$\mathbf{R}^{(j)}$ : IF $x_{1}$ is $F_{1}^{j}$ and $x_{n}$ is $F_{n}^{j}$, THEN $y^{j}$ is $C^{j}$, where $\boldsymbol{x}=\left[\begin{array}{ll}x_{1} & x_{2}\end{array}\right]^{\mathrm{T}}$ is the input vector and $\boldsymbol{y} \in \mathbf{R}$ is the output variable. By using the singleton fuzzification, product inference engine and center average defuzzification, the output value of the fuzzy system is given as

$$
y(x)=\frac{\sum_{j=1}^{m} y^{j}\left(\prod_{i=1}^{n} \mu_{F_{i}^{j}}\left(x_{i}\right)\right)}{\sum_{j=1}^{m}\left(\prod_{i=1}^{n} \mu_{F_{i}^{j}}\left(x_{i}\right)\right)}
$$

where $y^{j}$ represents a crisp value at which the output membership function $\mu_{F_{i}^{j}}$ reaches its maximum value, i.e., $\mu_{F_{i}^{j}}\left(y^{j}\right)=1$.

The fuzzy PID controller is based on the conventional PID controller, using the fuzzy set to impress the control rules' conditions and operation. The output of the PID controller is the main output of the Fuzzy PID controller, and the output of the fuzzy controller is to make some corresponding adjustments on the base of the output of the PID controller. The control structure is shown in Fig. 4.

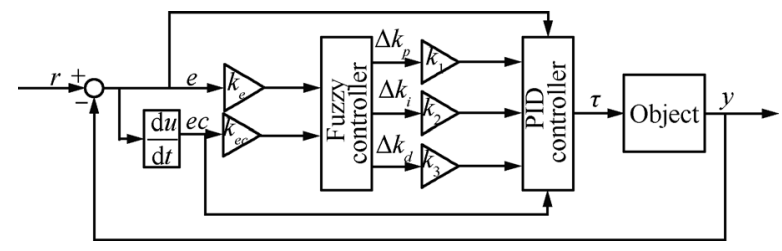

Fig. 4 Fuzzy PID control structure

In this paper the input $r$ in Fig. 4 denotes the desired joint position $q_{d}$ obtained by inverse kinematics and $y$ is the actual joint position of the system obtained by dynamic analysis. The input variables of the fuzzy controller are the error $(e)$ between the desired joint position and the actual joint position and its change rate $(e c)$, while the output fuzzy variables are the adjustment parameters to the PID, namely $\Delta k_{p}, \Delta k_{i}$ and $\Delta k_{d}$, respectively. $k_{e}$ and $k_{e c}$ are blurring factors and $k_{1}, k_{2}$ and $k_{3}$ are solution fuzzy factors. Assume that $k_{p}^{0}, k_{i}^{0}$ and $k_{d}^{0}$ are the initial design values of the PID controller which are set by conventional method. The fuzzy PID parameters can be calculated as

$$
\left\{\begin{array}{l}
k_{p}=k_{p}^{0}+\Delta k_{p} k_{1} \\
k_{i}=k_{i}^{0}+\Delta k_{i} k_{2} \\
k_{d}=k_{d}^{0}+\Delta k_{d} k_{3} .
\end{array}\right.
$$

Compared with the conventional PID control method, this method does not depend on precise mathematics model and can adjust PID parameters on line in time. At the same time, it can achieve a better control effect.

The object in Fig. 4 denotes the dynamic models of the manipulator that have been derived from (3) and (6), and the fuzzy controller will be designed in the section below.

The membership functions of input and output linguistic variables as shown in Figs. 5 and 6 are decomposed into seven fuzzy partitions expressed as NB, NM, NS, ZO, PS, $\mathrm{PM}, \mathrm{PB}$. The structure of the selected fuzzy controller is shown in Fig. 7.

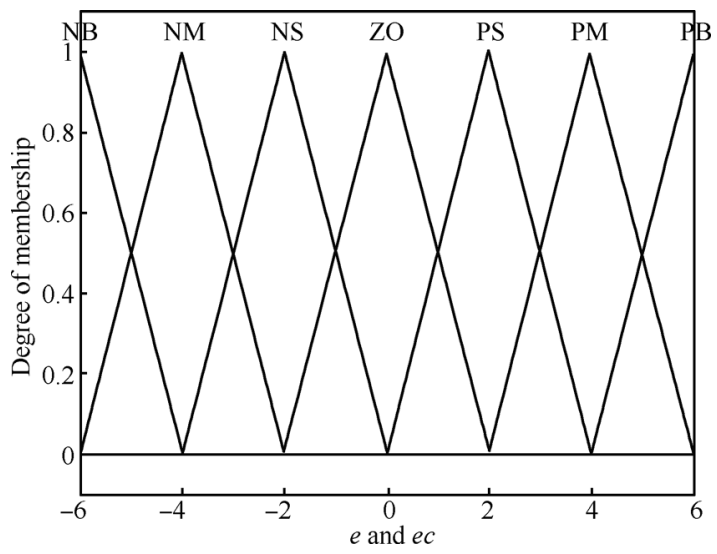

Fig. 5 The membership functions of input variables

The fuzzy rules are extracted in such a way that the system must be stable and these rules contain the input-output relationships that define the control strategy. For the fuzzy implication, the intersection minimum operation has been used, the gravity method defuzzification process has been selected. On the basis of the experience of [16], and taking 
account of the actual control system of this paper, the fuzzy rule tables are designed as in Tables 1-3. When we get the fuzzy rule we can take full use of the Matlab fuzzy control toolbox to input the corresponding rules.

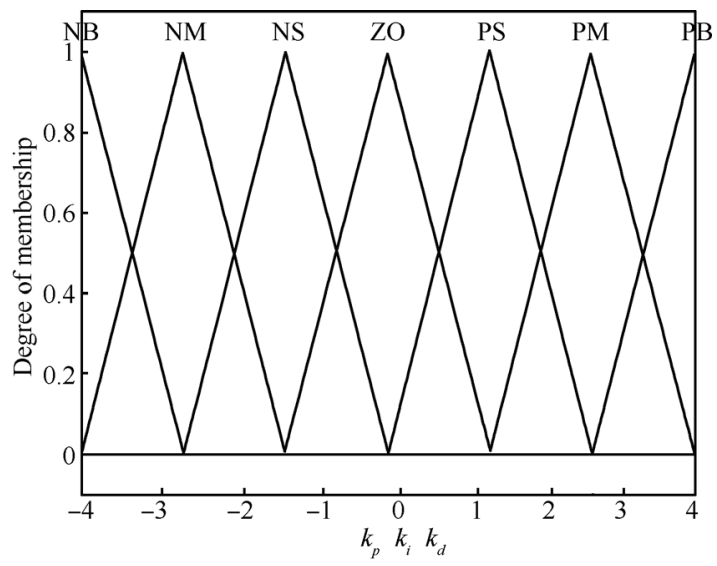

Fig. 6 The membership functions of output variables

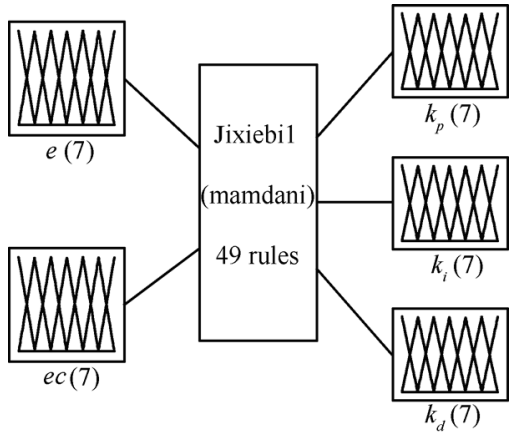

System jixiebi1: 2 inputs, 3 outputs, 19 rules

Fig. 7 Fuzzy controller structure

Table 1 Fuzzy rule base of $k_{d}$

\begin{tabular}{cccccccc}
\hline & \multicolumn{7}{c}{$e c$} \\
\cline { 2 - 7 }$e$ & NB & NM & NS & ZO & PS & PM & PB \\
\hline NB & PS & PS & PM & PM & PS & ZO & ZO \\
NM & PS & PB & PM & PS & PS & ZO & NS \\
NS & PM & PM & PM & PS & ZO & NS & NS \\
ZO & PM & PM & PS & ZO & NS & NM & NM \\
PS & PS & PS & ZO & NS & NS & NM & NM \\
PM & PS & ZO & NS & NM & NM & NM & NB \\
PB & ZO & ZO & NM & NM & NM & NB & NB \\
\hline
\end{tabular}

Table 2 Fuzzy rule base of $k_{i}$

\begin{tabular}{cccccccc}
\hline \multirow{2}{*}{$e$} & \multicolumn{7}{c}{$e c$} \\
\cline { 2 - 7 } & NB & NM & NS & ZO & PS & PM & PB \\
\hline NB & NB & NB & NM & NM & NS & ZO & ZO \\
NM & NB & NB & NM & NS & NS & ZO & ZO \\
NS & NB & NM & NS & NS & ZO & PS & PS \\
ZO & NB & NB & NS & ZO & PS & PM & PM \\
PS & NM & NM & ZO & PS & PS & PM & PB \\
PM & NS & NS & PS & PS & PM & PB & PB \\
PB & ZO & ZO & PS & PM & PM & PB & PB \\
\hline
\end{tabular}

Table 3 Fuzzy rule base of $k_{p}$

\begin{tabular}{cccccccc}
\hline & \multicolumn{7}{c}{$e c$} \\
\cline { 2 - 7 }$e$ & NB & NM & NS & ZO & PS & PM & PB \\
\hline NB & PB & PS & NB & NM & NB & NM & PS \\
NM & PB & PB & NB & NM & NM & NS & ZO \\
NS & ZO & NS & NM & NM & NS & NS & ZO \\
ZO & ZO & NS & NS & NS & NS & NS & ZO \\
PS & ZO & ZO & ZO & ZO & ZO & ZO & ZO \\
PM & PB & NS & PS & PS & PS & PS & PB \\
PB & PB & PM & PM & PM & PS & PS & PB \\
\hline
\end{tabular}

\section{Simulation research}

The fuzzy PID controller is now examined for its ability to control the space manipulator to track a given trajectory for both ground alignment and space applications. Simulations are carried out on a two-link space manipulator as shown in Figs. 2 and 3. The time taken is $10 \mathrm{~s}$. The time step for the computer simulation is $0.01 \mathrm{~s}$. The majority of the simulations are about the angle tracking ${ }^{[9,20-26]}$. It cannot see how the end-effector moves. In this paper, the end-effector is required to track a desired trajectory according to inverse kinematics. The desired trajectory of the end-effector is a circle that can be seen from (9). The trajectory tracking control framework is shown in Fig. 8, from which we can see that the desired joint position and the control torque are obtained by inverse kinematics and dynamic analysis of the manipulator. The parameters of the space manipulator used for simulation are shown in Table 4.

$$
\left\{\begin{array}{l}
x_{d}=0.28 \cos \left(\frac{\pi t}{5}\right)+0.85 \\
y_{d}=0.28 \sin \left(\frac{\pi t}{5}\right)
\end{array}\right.
$$

Table 4 Two-link manipulator parameters

\begin{tabular}{ccccc}
\hline link & $a_{i}(\mathrm{~m})$ & $b_{i}(\mathrm{~m})$ & $m_{i}(\mathrm{~kg})$ & $I_{i}\left(\mathrm{~kg} \cdot \mathrm{m}^{2}\right)$ \\
\hline 0 & - & 0.5 & 40 & 6.667 \\
1 & 0.5 & 0.5 & 4 & 0.333 \\
2 & 0.5 & 0.5 & 3 & 0.25 \\
\hline
\end{tabular}

The simulation results of the proposed method that are compared with the corresponding conventional PID controller that contains a gravity compensation term which is equal to the gravity vector computed from formulas (3) and (6), and the control torques are computed as $\tau=k_{p} e+k_{i} \int e+k_{d} \dot{e}+G(q)$.

The controller parameters of PID are chosen as $k_{p}^{0}=250$, $k_{i}^{0}=1, k_{d}^{0}=50$; the blurring factors are $k_{e}=10, k_{e c}=1$; the solution fuzzy factor are $k_{1}=0.1, k_{2}=20, k_{3}=1$. When the space manipulator is controlled by the same PID controller for both ground alignment and space application the simulation results can be seen in Figs. 9 and 10.

It is obvious from Figs. 9 and 10 that the results obtained from Fig. 9 show better effect when compared with those from Fig. 10. It can be seen that the controller cannot drive the joints to reach the desired positions and there exists a steady-state tracking error. This is understandable since 


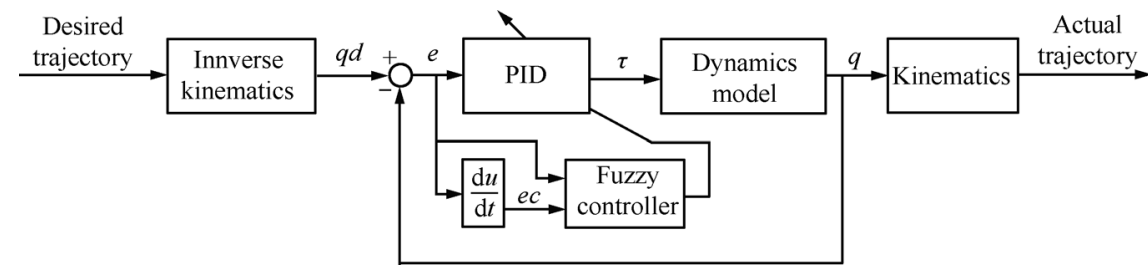

Fig. 8 Trajectory tracking control framework
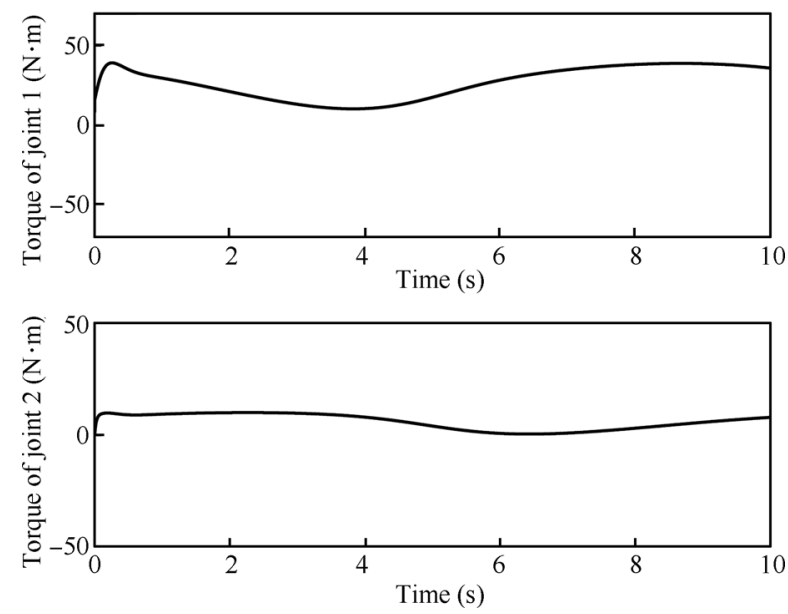

(a) Torques of joints 1 and 2

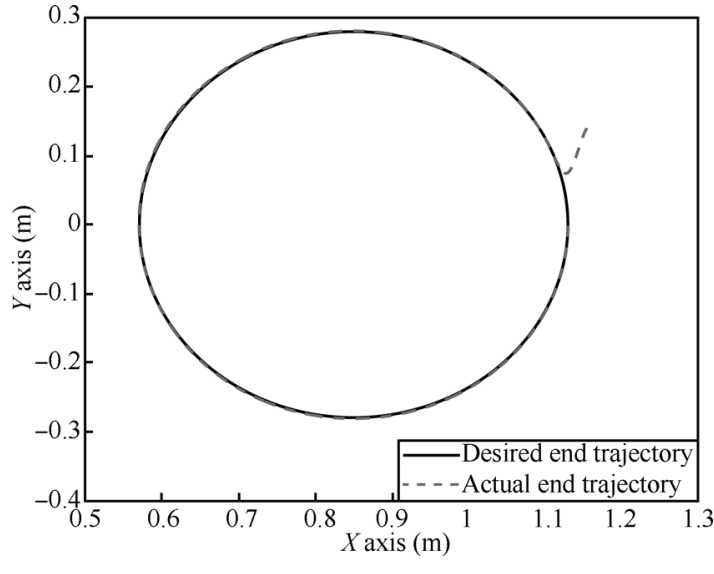

(b) End-effector trajectory tracking

Fig. 9 PID control trajectory tracking on the ground
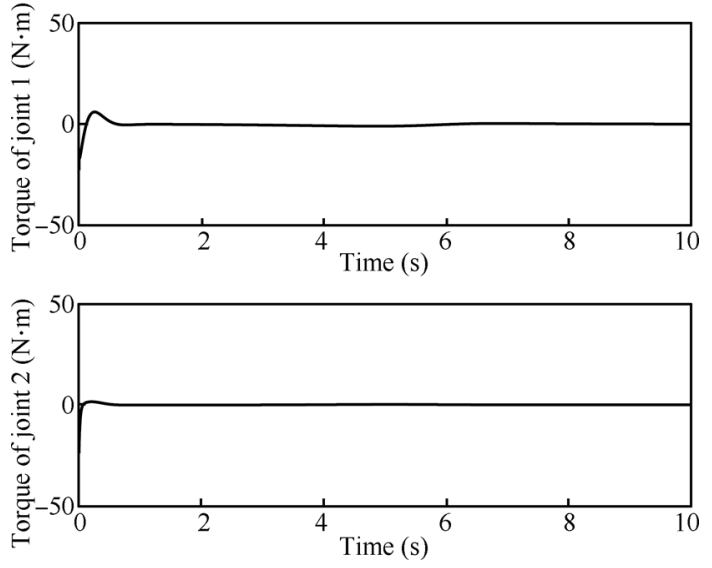

(a) Torques of joints 1 and 2

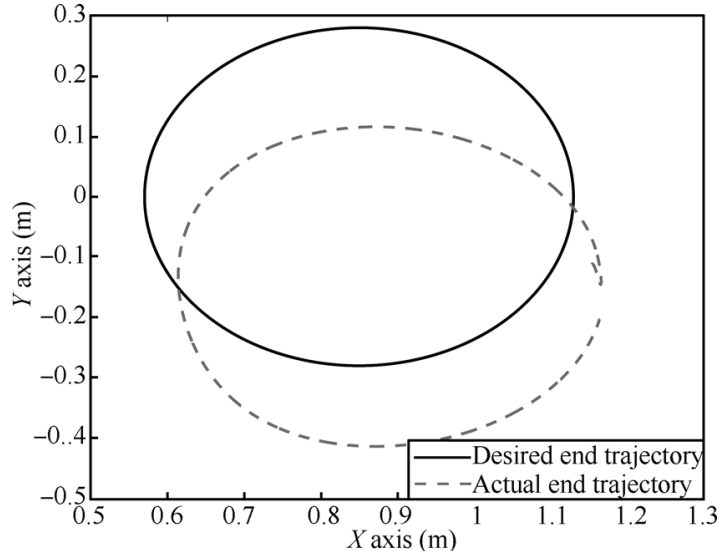

(b) End-effector trajectory tracking

Fig. 10 PID control trajectory tracking in space

the gravity will change from ground alignment to space applications. When the space manipulator enters into the space the gravity compensation term in the controller has an influence on the space manipulator so that the manipulator cannot track the desired trajectory well.

As stated in the previous section, the fuzzy PID controller has its advantage. Here, a fuzzy PID controller is adopted whose parameters have been stated in the previous section. When the space manipulator is in space, the PID parameters will be tuned by the fuzzy controller which is shown in Fig. 11. Figs. 12 and 13 show the trajectory tracking results with the fuzzy PID control for the two con- ditions.

Figs. 12 and 13 show the simulation results of the proposed fuzzy PID controller. It is observed that the control system can get a good tracking performance and the tracking errors converge to small values because of the on-line learning of the fuzzy logic system. The simulation results thus demonstrate the proposed fuzzy PID control can effectively control the system with model changes. Simulation results also show that the torque on the ground is bigger than that in the space. This is because the torque is relevant to the gravity. To validate the correctness of the conclusion, in this paper, $g$ is changed to observe the simulation results. 
Figs. 14 and 15 show the trajectory tracking results when $g$ is $4.9 \mathrm{~m} / \mathrm{s}^{2}$ and $1.0 \mathrm{~m} / \mathrm{s}^{2}$, respectively.
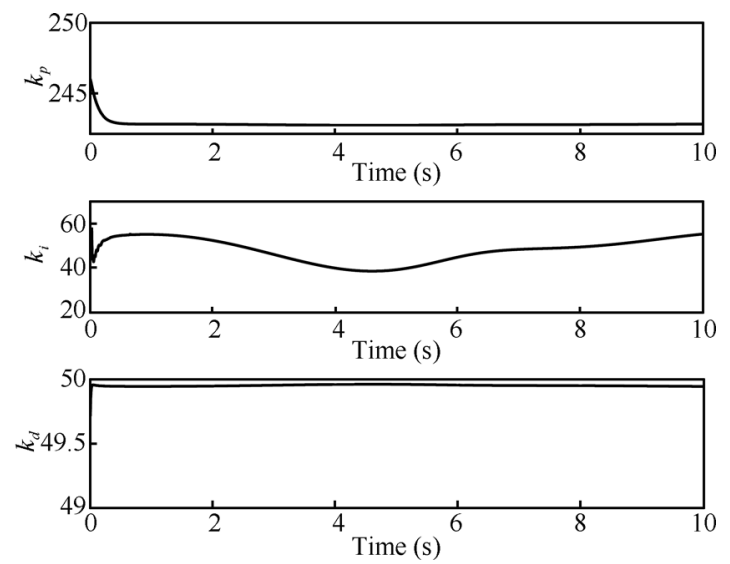

Fig. 11 PID parameters tuned by fuzzy controller
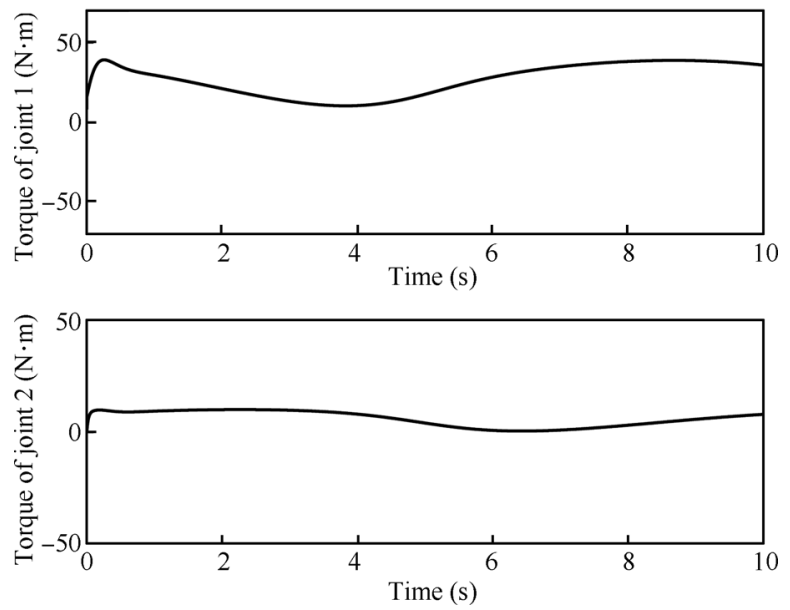

(a) Torques of joints 1 and 2

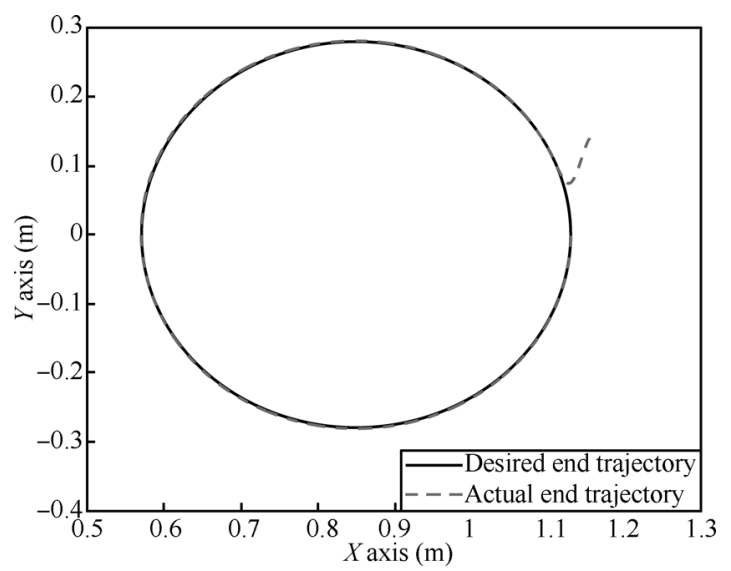

(b) End-effector trajectory tracking

Fig. 12 Fuzzy PID control trajectory tracking on the ground

As expected, smaller torques are obtained for a smaller gravitational acceleration $g$, as shown in Figs. 14 and 15 .
This demonstrates that the gravity does have influences on the torques. Controlled with the fuzzy PID, the system can get good control performance with a varied $g$. Simulation results demonstrate that the proposed adaptive fuzzy PID controller can effectively control the space manipulator system with model and parameters changing.
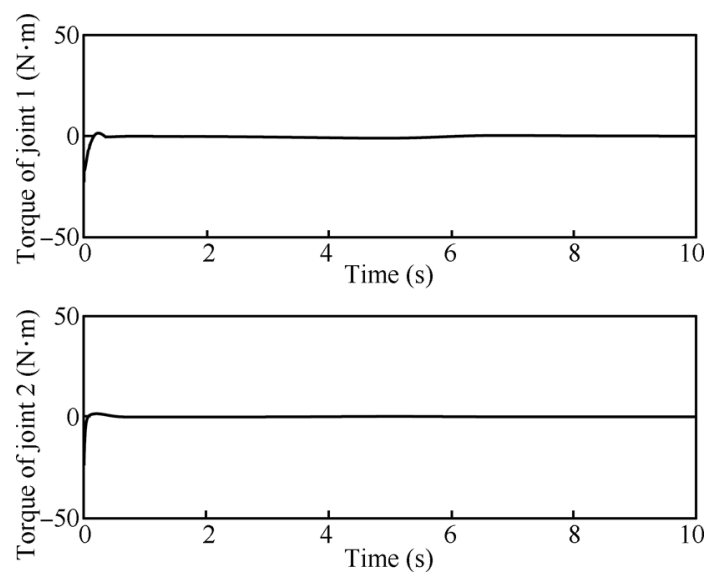

(a) Torques of joints 1 and 2

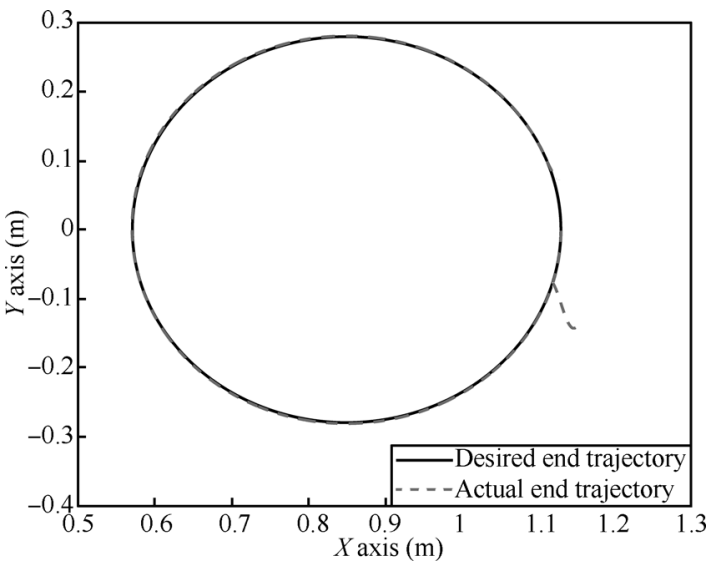

(b) End-effector trajectory tracking

Fig. 13 Fuzzy PID control trajectory tracking in space

\section{Conclusions}

To solve the model change problem when the space manipulator is aligned on the ground and applied in the space, this paper has established the dynamic models of the two cases. A fuzzy PID control scheme for space manipulator trajectory tracking control has been designed. Compared with the conventional PID control, simulation results show that the fuzzy PID controller derived in this paper demonstrates a better performance than the conventional PID controller. The proposed control algorithm is appropriate for practical control design robotic manipulator with model change and has important values for theoretical research and engineering application. 

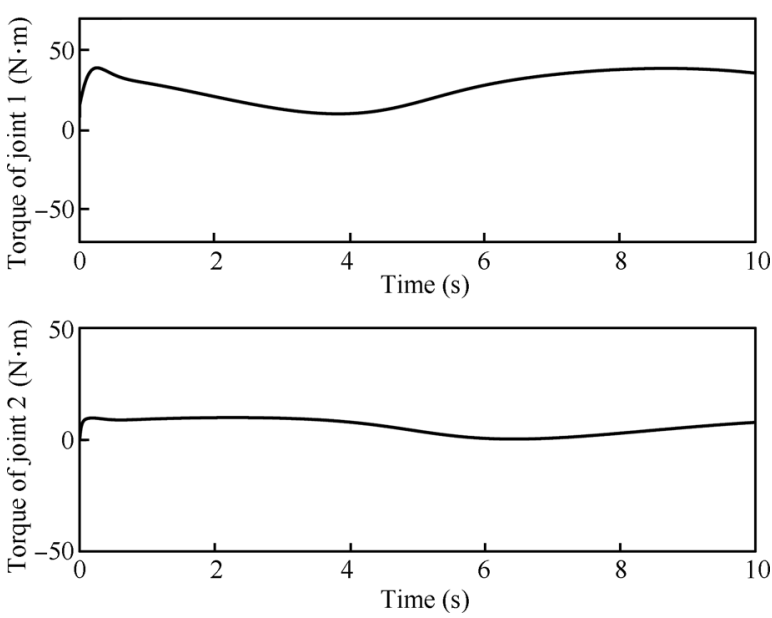

(a) Torques of joints 1 and 2

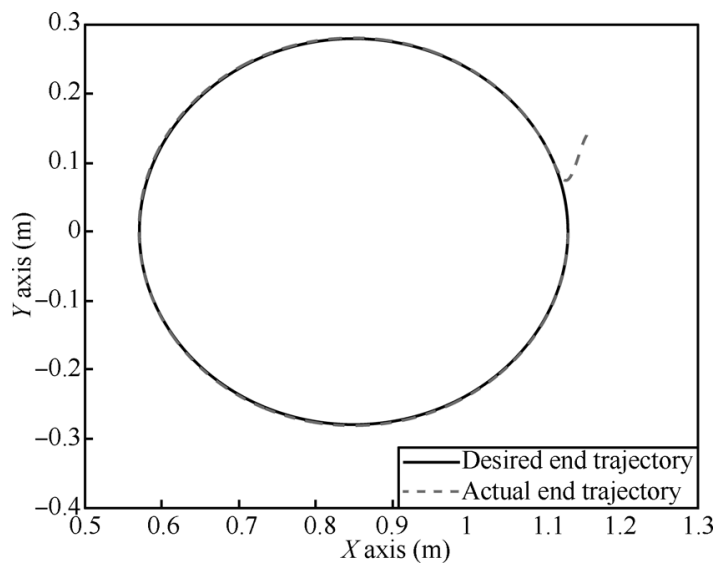

(b) End-effector trajectory tracking

Fig. 14 Fuzzy PID control trajectory tracking when $g=4.9 \mathrm{~m} / \mathrm{s}^{2}$
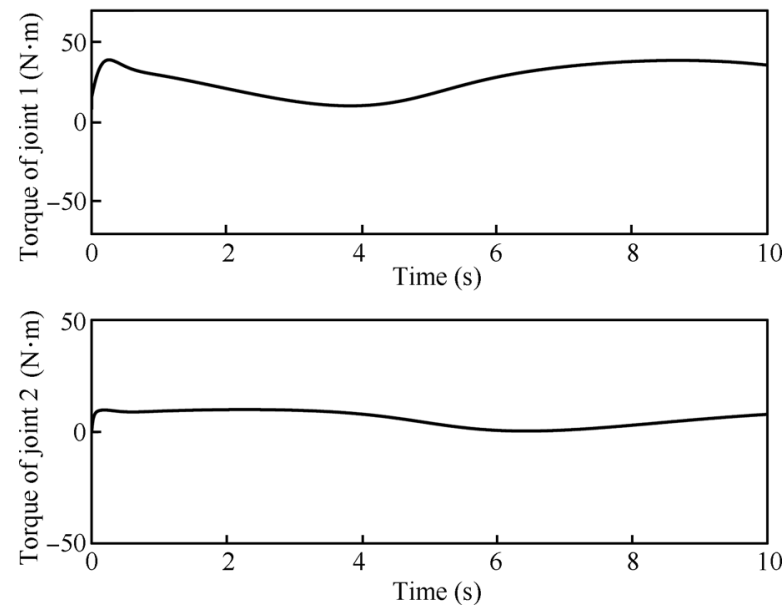

(a) Torques of joints 1 and 2

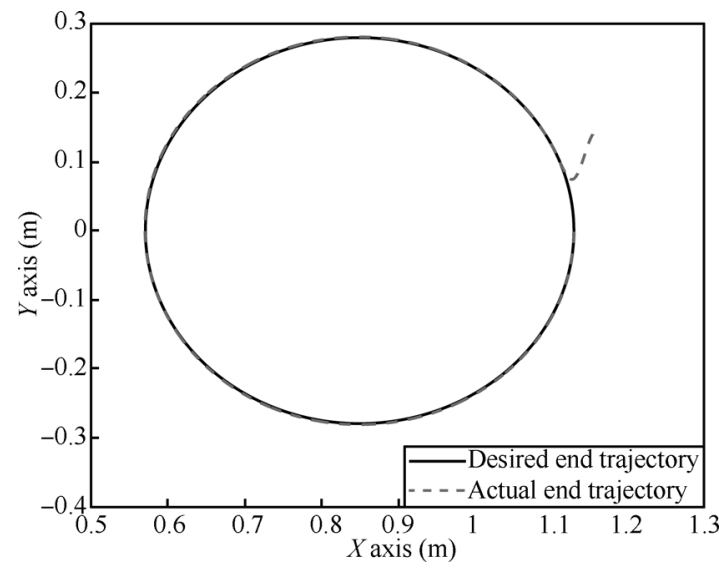

(b) End-effector trajectory tracking

Fig. 15 Fuzzy PID control trajectory tracking when $g=1.0 \mathrm{~m} / \mathrm{s}^{2}$

\section{Acknowledgement}

The authors would like to thank their research group for the help in the entire research process.

\section{References}

[1] E. Papadopoulos, S. Dubwsky. On the nature of control algorithms for free-floating space manipulators. IEEE Transactions on Robotics and Automation, vol. 7, no. 6, pp. 750$758,1991$.

[2] S. Purwar. Higher order sliding mode controller for robotic manipulator. In Proceedings of the 2007 IEEE International Symposium on Intelligent Control, IEEE, Singapore, pp. 556-561, 2007.

[3] A. Green, J. Z. Sasiadek. Fuzzy and optimal control of a two-link flexible manipulator. In Proceedings of the IEEE/ASME International Conference on Advanced Intelligent Mechatronics, IEEE, Como, pp. 1169-1174, 2001.

[4] E. A. M. Cruz, A. S. Morris. Fuzzy-GA-based trajectory planner for robot manipulators sharing a common workspace. IEEE Transactions on Robotics, vol.22, no. 4, pp. 613-624, 2006.
[5] L. Peng, P. Y. Woo. Neural-fuzzy control system for robotic manipulators. IEEE Control Systems Magazine, vol. 22, no. 1, pp. 53-63, 2006.

[6] Y. Zhao, C. C. Cheah, J. J. E. Slotine. Adaptive vision and force tracking control of constrained robots with structural uncertainties. In Proceedings of IEEE International Conference on Robotics and Automation, IEEE, Roma, pp. 23492354, 2007.

[7] H. L. Wang, Y. C. Xie. Passivity based adaptive Jacobian tracking for free-floating space manipulators without using spacecraft acceleration. Automatica, vol. 45, no. 6 , pp. 1510-1517, 2009.

[8] O. Parlaktuna, M. Ozkan. Adaptive control of free-floating space manipulators using dynamically equivalent manipulator model. Robotics and Autonomous Systems, vol. 46, no. 3, pp. 85-193, 2004.

[9] H. F. Ho, Y. K. Wong, A. B. Rad. Robust fuzzy tracking control for robotic manipulators. Simulation Modeling Practice and Theory, vol. 15, no. 7, pp. 801-816, 2007.

[10] H. R. Berenji, P. Khedkar. Learning and tuning fuzzy logic controllers through reinforcements. IEEE Transactions on Neural Networks, vol. 3, no. 5, pp. 724-740, 1992.

[11] R. E. Precup, S. Preitl, G. Faur. PI predictive fuzzy controllers for electrical drive speed control: Methods and 
software for stable development. Computers in Industry, vol. 52, no. 3, pp. 253-270, 2003.

[12] J. K. Liu. Intelligent Control, Beijing: Electronic Industry Press, pp. 48-51, 2009. (in Chinese)

[13] J. Carvajal, G. R. Chen, H. Ogmen. Fuzzy PID controller: Design, performance evaluation, and stability analysis. Information Sciences, vol. 123. no. 3-4, pp. 249-270, 2000.

[14] Z. Bingul, O. Karahan. A fuzzy logic controller tuned with PSO for 2 DOF robot trajectory control. Expert Systems with Applications, vol. 38, no. 1, pp. 1017-1031, 2011.

[15] Z. J. Sun, R. T. Xing, C. S. Zhao, W. Q. Huang. Fuzzy auto-tuning PID control of multiple joint robot driven by ultrasonic motors. Ultrasonics, vol. 46, no. 4, pp. 303-312, 2007.

[16] Y. H. Yin, S. K. Fan, M. E. Chen. The design and simulation of adaptive fuzzy PID controller. Fire Control and Command Control, vol. 33, no. 7, pp. 96-99, 2008. (in Chinese)

[17] S. B. Niku. Introduction to Robotics: Analysis, Systems, Applications, Beijing: Electronic Industry Press, pp. 107127, 2004. (in Chinese)

[18] J. J. Craig. Adaptive Control of Mechanical Manipulators, Reading, MA: Addison-Wesley, 1988.

[19] C. Harris, C. Moore, M. Brown. Intelligent Control: Aspects of Fuzzy Logic and Neural Nets, River Edge, NJ: World Scientific, 1993.

[20] M. Boukattaya, M. Jallouli, T. Damaka. On trajectory tracking control for nonholonomic mobile manipulators with dynamic uncertainties and external torque disturbances. Robotics and Autonomous Systems, vol.60, no. 12, pp. 1640-1647, 2012.

[21] I. Cervantesa, J. Alvarez-Ramirezb. On the PID tracking control of robot manipulators. Systems and Control Letter, vol. 42, no. 1, pp. 37-46, 2001.

[22] H. L. Wang. On adaptive inverse dynamics for free-floating space manipulators. Robotics and Autonomous Systems, vol. 59, no. 10, pp. 782-788, 2011.

[23] J. C. Liu, Y. Miao. Research on trajectory control strategy of robot arm based on neural network compensation. Control and Decision, vol. 20, no. 7, pp. 732-736, 2005. (in Chinese)
[24] X. D. Zhang, Q. X. Jia, H. X. Sun, M. Chu. The research of space robot flexible joint trajectory control. Journal of Astronautics, vol. 29, no. 6, pp. 1865-1869, 2008. (in Chinese)

[25] Y. S. Guo, L. Chen. Adaptive neural network control of free-floating space manipulator system in joint space. In Proceedings of the 26th Chinese Control Conference, IEEE, Hunan, China, pp. 117-120, 2007.

[26] L. P. Xi, H. He, H. R. Dong. Method of chattering elimination in sliding mode tracking control of robotic manipulators. Computer Simulation, vol. 29, no. 5, pp. 188-191, 2012. (in Chinese)

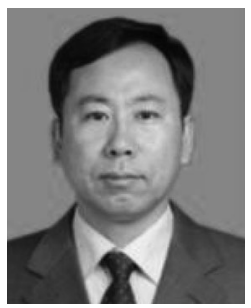

Fu-Cai Liu graduated from Harbin Institute of Technology, China in 2003. He received the M. Sc. degree of electric drive and automation from the Northeast Heavy Machinery Institute, China in 1994 and the $\mathrm{Ph}$. D. degree of control theory and control engineer from Harbin Institute of Technology in 2003. He is currently a professor in Yanshan University, China.

His research interests include fuzzy identification and control and space manipulator control.

E-mail: lfc@ysu.edu.cn (Corresponding author)

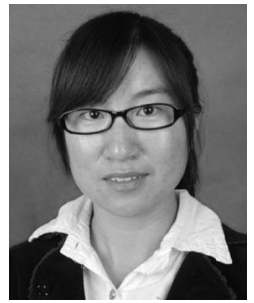

Li-Huan Liang graduated from Yanshan University, China in 2012. She received the bachelor's degree of automation from Yanshan University, China in 2012. She is currently a postgraduate in Yanshan University, China.

Her research interests include robot control technologies.

E-mail: lhliang719@163.com

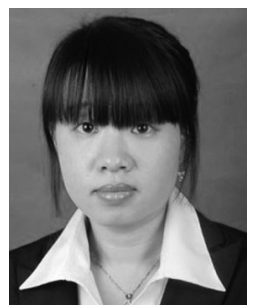

Juan-Juan Gao graduated from Yanshan University, China in 2013. She received her M.Sc. degree from Institute of Electrical Engineering, Yanshan University, China in 2013.

Her research interests include space manipulator modeling and control.

E-mail: gaojuanjuan_2008@163.com 